\title{
Higher Titer of TSH Receptor Antibody Was Associated with Better Responsiveness of Thyroid Hormone to Antithyroidal Drug in Graves' Disease.
}

${ }^{1}$ Hoon Sung Choi, ${ }^{2}$ Won Sang Yoo

1 Department of Internal Medicine, Kangwon National University Hospital, Chuncheon-si, Republic of Korea 2 Department of Internal Medicine, Dankook University Hospital, Cheonan-si, Republic of Korea

\section{Background}

- Graves' disease is a common autoimmune disease caused by autoantibody to TSH receptor (TSHRAb), and Anti-thyroidal drug (ATD) therapy is considered as choice of treatment for Graves' disease. Although ATD therapy generally shows good efficacy to mitigate thyrotoxic symptoms and normalize thyroid function, the responsiveness varies.

- Previous studies suggested young age, male gender, severe thyrotoxicosis at diagnosis and high titer of TSHRAb as risk factors for poor responsiveness to ATD treatment; however, clinical guidelines did not yet recommend the optimized treatment protocols.

- In this study, we aimed to examine the factors associated with responsiveness to ATD treatment.

\section{Methods}

- Our study included 94 patients with Graves' disease, whose TSHRAb titers were measured at diagnosis and whose free thyroxine were repetitively measured during follow-up.

- Responsiveness to ATD therapy was considered as correlation coefficients between exposed ATD dose and decreasing rates of free thyroxine levels. We analyzed the association between calculated responsiveness and clinical characteristics, including age, sex and TSHRAb titer.

- Using R packages, $\mathrm{P}$ values under 0.05 were considered as statistically significant.

\section{Results}

- Mean age $=47.1 \pm 15.4$ years old

- Male : Female $=26(28 \%): 68(72 \%)$

- Mean value of TSHRAb titer $=19.1 \pm 14.0 \mathrm{IU} / \mathrm{L}$

Figure 1. Comparison of responsiveness among subgroups, according to age, sex and TSHRAb titer

(A) Age

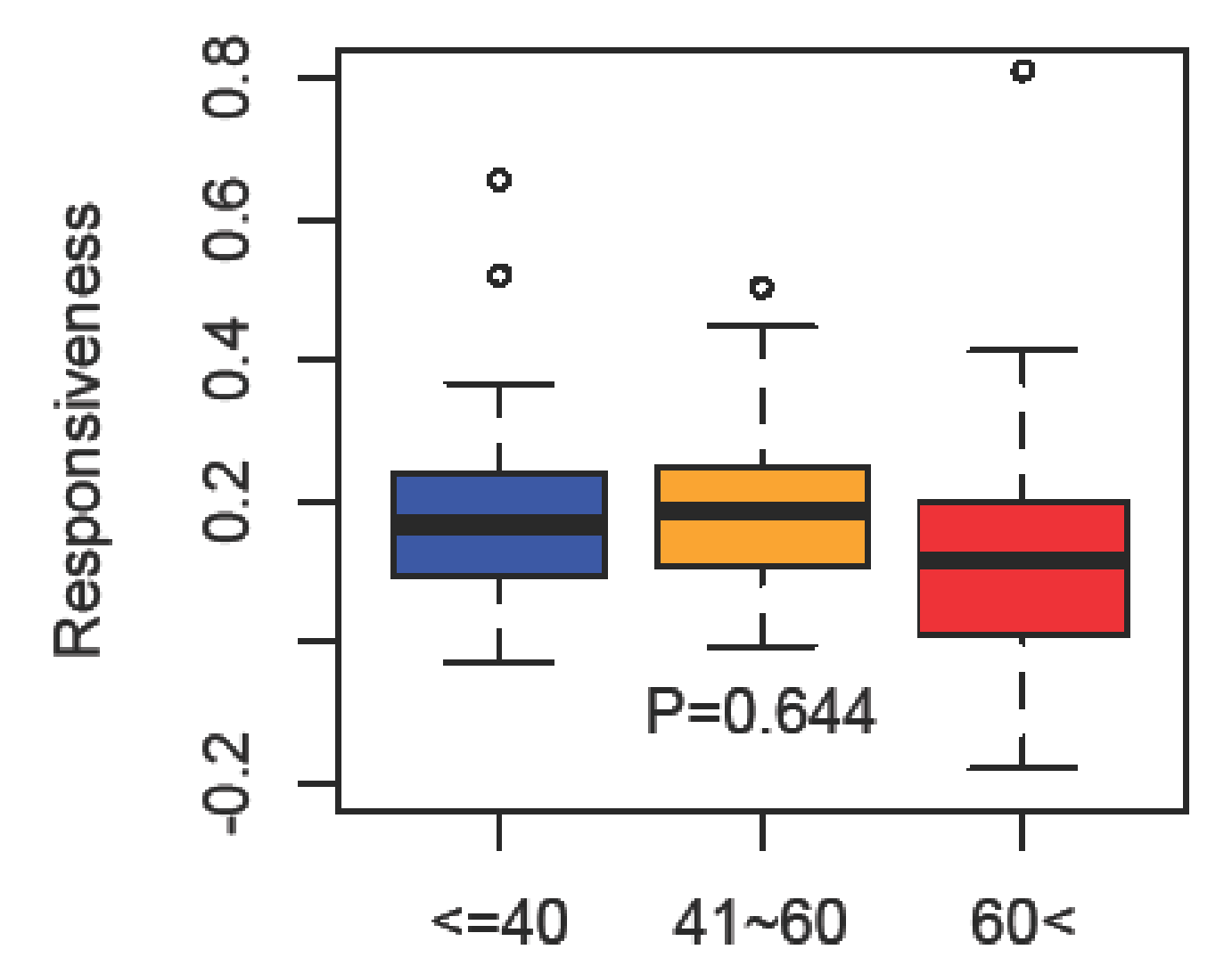

(B) Sex

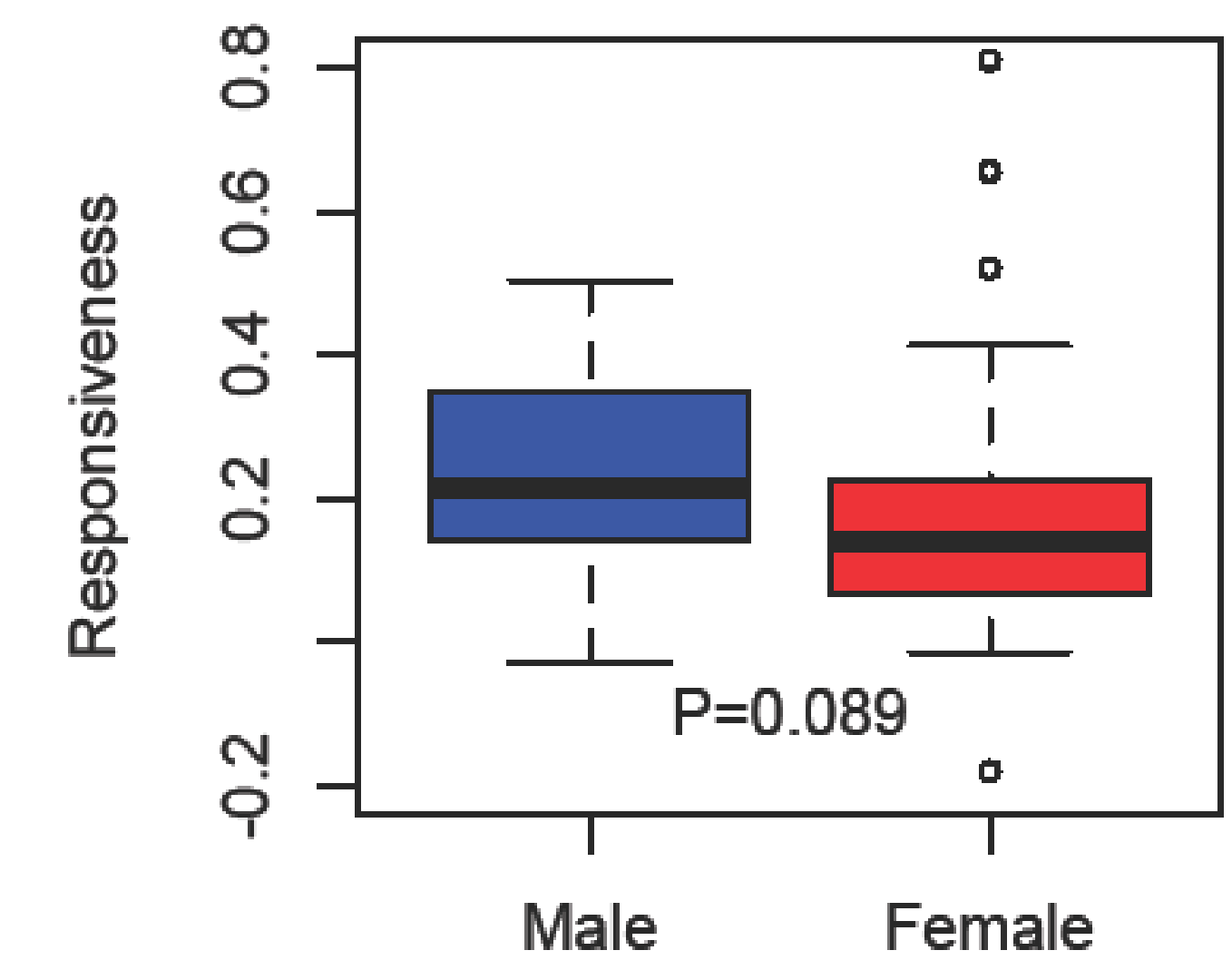

(C) TSHRAb titer

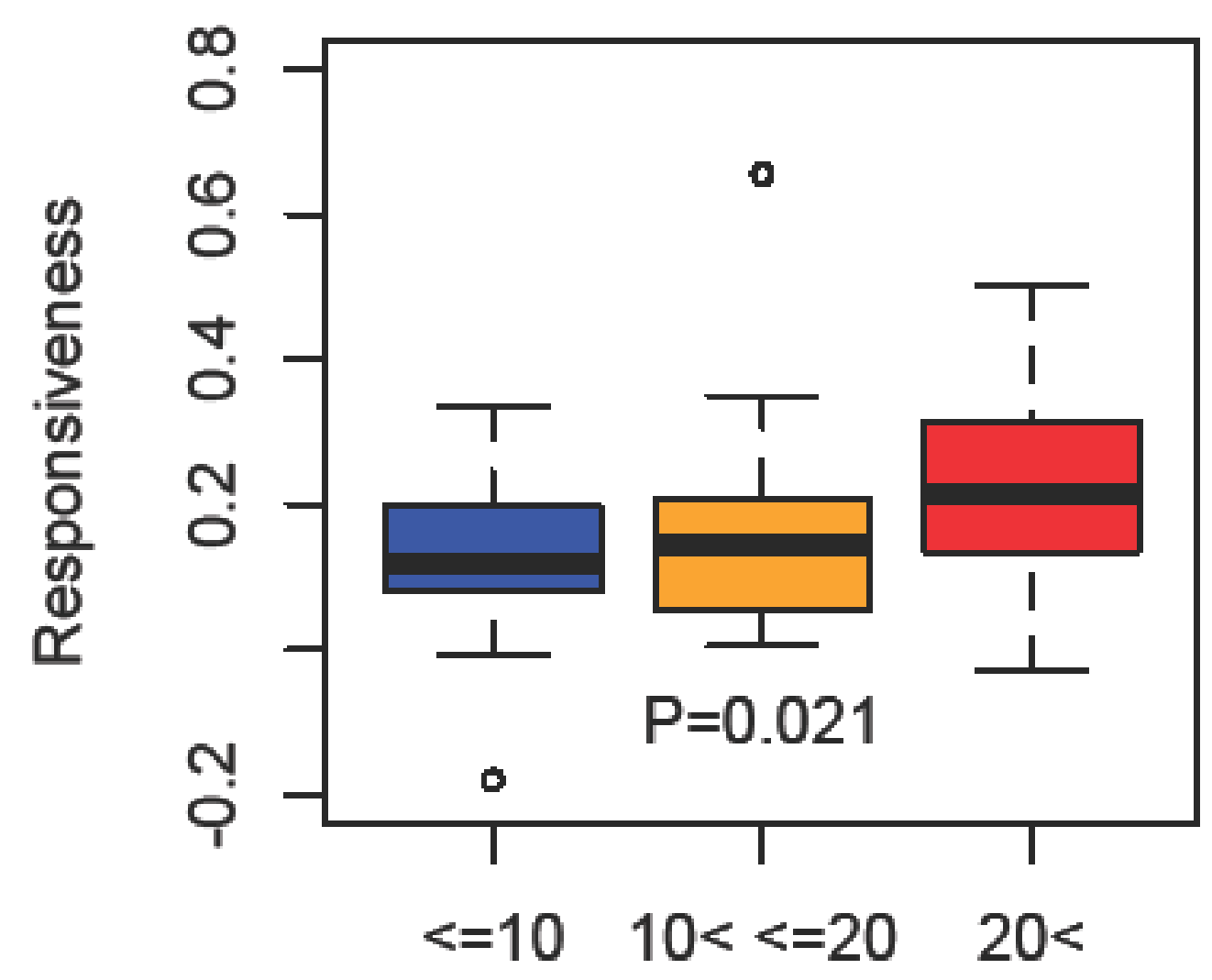

Figure 2. Association between age and responsiveness to ATD treatment

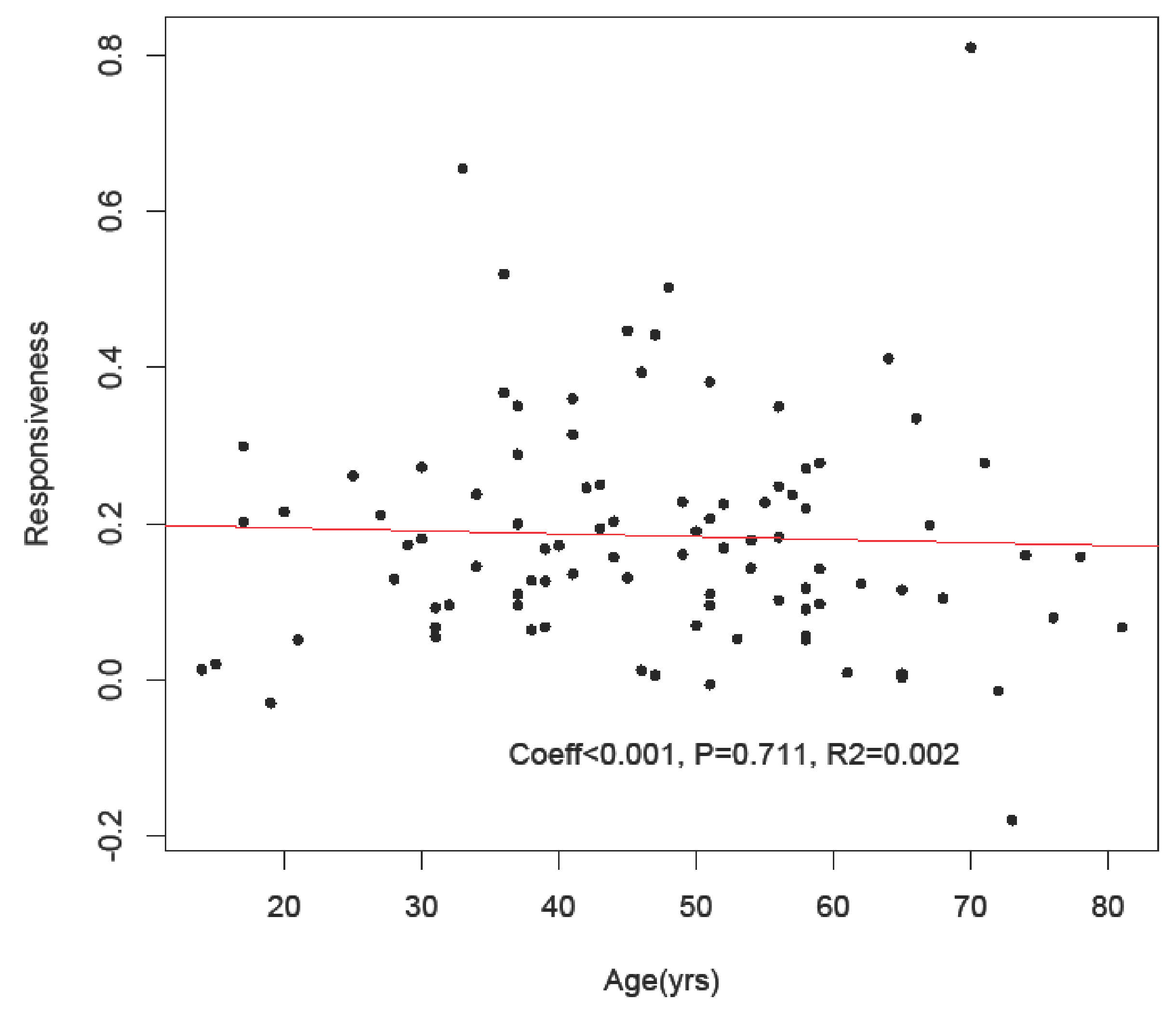

Figure 3. Association between TSHRAb titer and responsiveness to ATD treatment

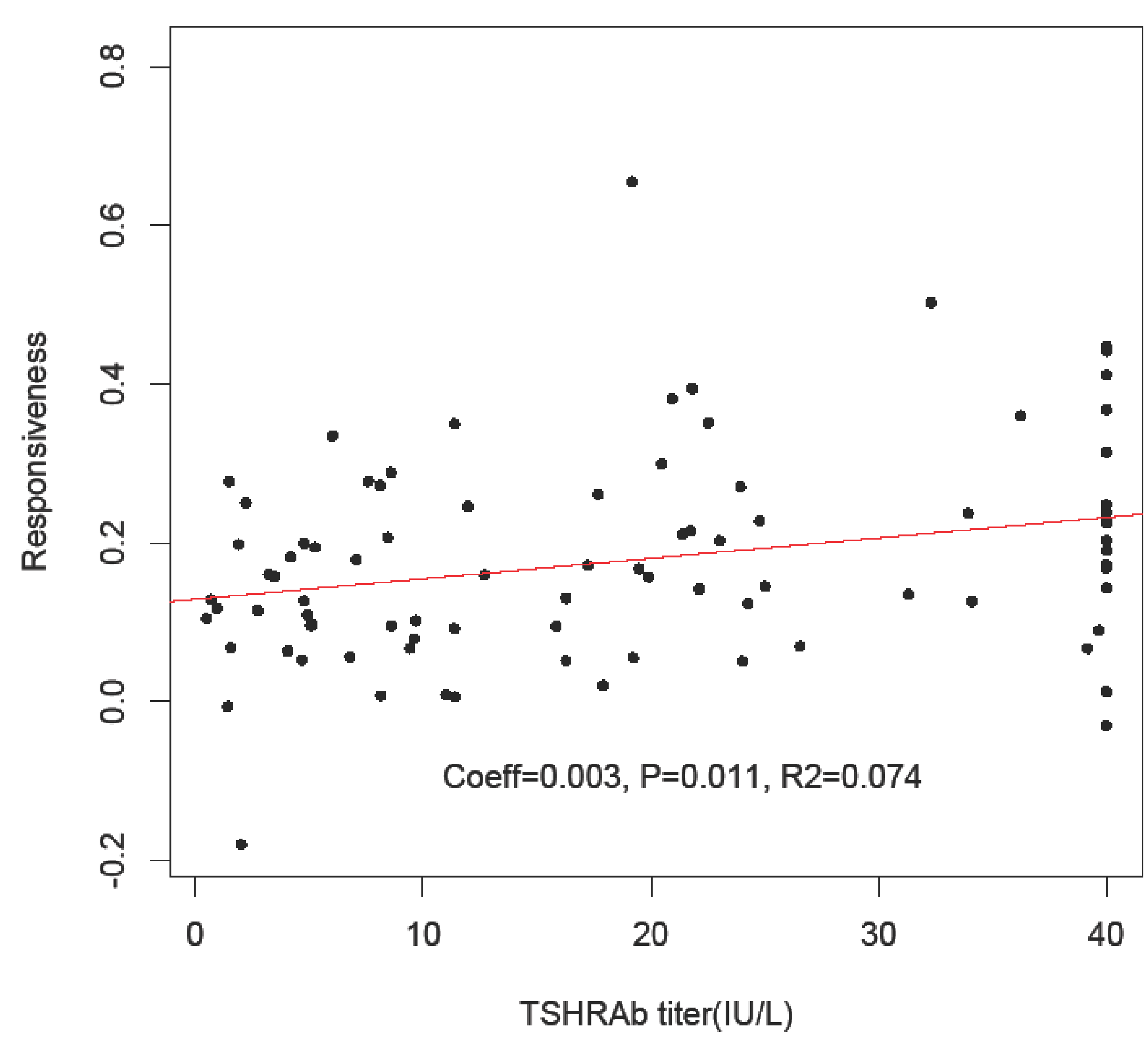

Table 1. Multivariate analyses for association between responsiveness to ATD and factors, adjusted for age and sex

\begin{tabular}{|l|c|c|c|}
\hline & Coefficiencts & SE & P value \\
\hline Age (yrs) & $<0.001$ & $<0.001$ & 0.920 \\
\hline Female & -0.061 & 0.003 & 0.047 \\
\hline TSHRAb titer & 0.002 & $<0.001$ & 0.021 \\
\hline
\end{tabular}

\section{Summary}

- Responsiveness to ATD, which was defined as correlation coefficients between decreasing rates of free thyroxine levels and exposed ATD dose, showed significant positive correlation with TSHRAb titers.

- Significant association of TSHRAb titer remained in multivariate analyses, with adjustment for age and sex.

- Our results suggest the usefulness of TSHRAb titer to decide the optimal initial ATD dose and to estimate responsiveness of individual patients during treatment periods. 